\title{
Contribution à l'étude d'une ressource naturelle renouvelable la pêche professionnelle en eau douce en France
}

\section{Didier Babin}

Abstract

Contribution to a renewable natural resource study : small-scale inland fischeries in France Concepts and pratices of farming system study are applied tofrench small-scale inland fischeries. The systemic approach is used to develop tools for acquisition, synthesis and representation knowledges.

\section{Résumé}

Les concepts et pratiques de l'étude des systèmes d'exploitation agricole ont été adaptés à la pêche artisanale continentale française. Délibérément systémique, l'approche cherche à développer des outils d'acquisition, de synthèse et représentation des connaissances.

\section{Citer ce document / Cite this document :}

Babin Didier. Contribution à l'étude d'une ressource naturelle renouvelable la pêche professionnelle en eau douce en France . In: Économie rurale. $\mathrm{N}^{\circ} 222,1994.48$ heures - 48 thèses d'Économie et de Sociologie Rurales. [Session d'automne 1993 (9 et 10 novembre à l'École Nationale du Génie Rural des Eaux et des Forêts) ] p. 14;

doi : https://doi.org/10.3406/ecoru.1994.4921

https://www.persee.fr/doc/ecoru_0013-0559_1994_num_222_1_4921

Fichier pdf généré le 08/05/2018 


\section{CONTRIBUTION A L'ÉTUDE D'UNE RESSOURCE NATURELLE RENOUVELABLE LA PÊCHE PROFESSIONNELLE EN EAU DOUCE EN FRANCE}

\section{Didier BABIN}

Chercheur au CIRAD-Forêt/ GREENLESS

U.R. Gestion REssources REnouvelables ENvironnement

Laboratoire d'Economie et de Sciences Sociales

45 bis, avenue de la Belle-Gabrielle

94736 Nogent-sur-Marne Cedex

Thèse réalisée dans le cadre de l'Université de Haute-Bretagne, U.F.R. Géographie et Aménagement de l'Espace (Rennes : J.-P. Marchand) auprès du CEMAGREF de Bordeaux, Division Aquaculture et Pêche (Gazinet : P. Elie).

Résumé : Les concepts et pratiques de l'étude des systèmes d'exploitation agricole ont été adaptés à la pêche artisanale continentale française. Délibérement systémique, l'approche cherche à développer des outils d'acquisition, de synthèse et représentation des connaissances.

\section{CONTRIBUTION TO A RENEWABLE NATURAL RESOURCE STUDY : SMALL-SCALE INLAND FISCHERIES IN FRANCE}

\section{Summary : Concepts and pratices of farming system study are applied to french small-scale inland fischeries. The systemic} approach is used to develop tools for acquisition, synthesis and representation knowledges.

Dans le domaine scientifique, les "choses" touchant aux ressources renouvelables en général et à l'halieutique en particulier sont en pleine effervescence. On assiste au développement et à la diffusion d'approches relativement nouvelles pour l'étude des pêcheries dans une optique de compréhension ou d'aménagement.

\section{APPROCHE SYSTÉMIQUE DE LA PÊCHE \\ PROFESSIONNELLE EN EAU DOUCE}

L'organisation globale du secteur est liée aux interactions entre le pêcheur professionnel et son environnement. Des contraintes de cet environnement sur la pêche paraissent primordiales comme : (i) la pression juridico-administrative (statut du pêcheur, police de la pêche, attribution des droits de pêche, etc), (ii) la pression sociale (concurrence pour la ressource piscicole, conflit d'utilisation de l'espace, etc), (iii) la demande des consommateurs, (iv) un environnement physique et écologique incertain et risqué par sa double variabilité temporelle et spatiale, (v) un processus historique. Trois réponses de la communauté de pêcheurs paraissent fondamentales : (i) un savoir-faire pour adapter leurs pratiques aux contraintes physiques du milieu et aux particularités écologiques et biologiques des espèces recherchées, à la réglementation et aux attentes des consommateurs, (ii) la mise en place d'un modèle moderne du "pêcheur professionnel en eau douce", d'une lutte pour le contrôle de ce titre et d'une crise d'identité professionnelle, (iii) la recherche d'une reconnaissance sociale, économique et politique par un mouvement de professionnalisation.

Dans cet espace de libertés et de contraintes, chaque pêcheur professionnel développe ses projets en fonction de ses propres marges de manœuvre. L'unité de pêche est considérée comme un système où les aspects familiaux et le volet entreprise sont aussi pris en compte. Ainsi une unité dénommée "Système PêcheurEntreprise-Famille" (SPEF) est proposée.

\section{OBTENTION ET TRAITEMENT DE L'INFORMATION}

L'étude des systèmes Pêcheur-Entreprise-Famille a été réalisée à partir de données originales (enquêtes de terrain auprès de plus de 300 SPEF et mise en place d'un réseau national d'information et de suivi par enquêtes postales). Devant l'apparente hétérogénéité de ces SPEF, la réalisation d'une typologie a été entreprise (chaine typologique associant Analyse Factorielle Multiple de plusieurs sous-tableaux et Classification Ascendante Hiérarchique). Cinq facteurs principaux ont été retenus et analysés : (i) les phénomènes de pluri ou monoactivité et l'ancrage ou non dans la profession, (ii) l'isolement ou non des pêcheurs au niveau familial et professionnel, (iii) la rente de situation possible par héritage ou localisation, (iv) le degré de professionnalisme familial, (v) l'histoire halieutique des pêcheurs (itinéraire professionnel).

\section{RÉSULTATS}

Huit types de SPEF sont décrits (analyse socio-spatiale) : (i) les marins-pêcheurs terriens, (ii) la relève hésitante, voire opportuniste, (iii) les estuariens pluriactifs professionnalisés, (iv) les entreprises familiales de pêche et d'activités connexes, (v) les vieux agri-pêcheurs récemment professionnalisés, (vi) les systèmes "féminisés" et les méconnus, (vii) les vieilles familles des cours d'eau intérieurs et des lacs, (viii) les pêcheurs traditionnels des lacs alpins.

Une première évaluation socio-économique précise de l'activité a pu être réalisée pour l'année 1989. L'activité de pêche professionnelle en eau douce en France occupe, au niveau de la production, 464 SPEF (regroupant 715 personnes pêcheurs ou travailleurs de la pêche, à temps plein ou à temps partiel). La production représente environ 1500 tonnes pour un chiffre d'affaires (c.a.) à la première mise en marché d'environ 55 millions de francs ; 42 espèces de poissons, d'agnathes ou de crustacés sont produites ; 45 cours d'eau ou lacs sont exploités surtout sur la façade atlantique et le secteur rhône-alpin dans 34 départements au total. Au niveau national, le c.a. pour les espèces grandes migratrices (anguille, lamproies, aloses, mulets, saumon atlantique, flet) représente un peu plus de $60 \%$ du chiffre total ( $45 \%$ pour la seule espèce anguille). Celui des espèces du cortège faunistique des lacs alpins (corégones, perche, truite, omble chevalier, lotte) est de $15 \%$. Au niveau national un quart seulement du c.a. provient d'autres espèces (poissons blancs, friture, sandre, écrevisse, brochet, etc). Trois types essentiels de clients achètent les produits : grossistes et mareyeurs (54\% du c.a.), restaurateurs $(22 \%)$ et particuliers $(18 \%)$.

Grâce à l'atlas des pêcheries réalisé, une approche par espèce d'intérêt halieutique peut aussi être effectuée à différents niveaux (national, départemental ou par bassin ; répartition et proportion des SPEF s'intéressant à l'espèce ; productions par c.a. et par tonnage ; modes de commercialisation ; techniques de la pêcherie).

\section{AMÉNAGEMENTS SUGGÉRÉS ET CONCLUSIONS}

La connaissance des systèmes de pêche permet de mieux définir le degré d'acceptation ou d'adaptabilité de ces systèmes à des scénarii d'aménagement. Les suggestions d'aménagement vont dans le sens de la professionnalisation des pêcheurs observée lors de ce travail et d'une gestion à long terme du patrimoine piscicole. Trois niveaux complémentaires sont à prendre en compte : (i) un statut juridique, social et fiscal pour les entreprises de pêche qui pourrait servir aussi à d'autres types d'entreprises rurales, (ii) un système de formation des pêcheurs leur permettant d'acquérir ou de compléter leur capacité à manager leur projet d'entreprise, (iii) la mise en place d'un observatoire de l'activité et de la ressource par collaboration entre pêcheurs, scientifiques et administrations gestionnaires. 